
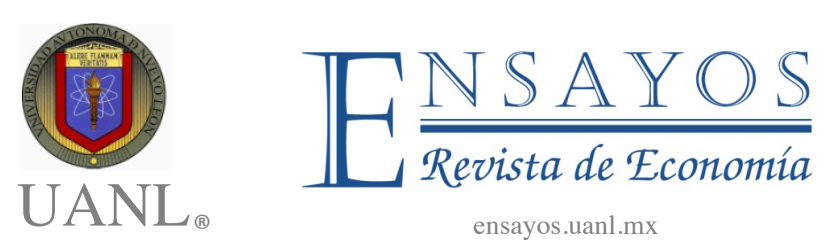

ensayos.uanl.mx

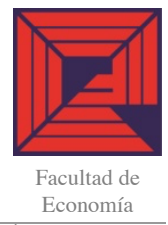

\title{
Desigualdad salarial en los subsectores manufactureros en México, 2007-2018
}

Wage Inequality in Mexico's Manufacture, 2007-2018

Brenda Murillo-Villanueva*
Yolanda Carbajal Suárez $^{* *}$
Leobardo de Jesús Almonte $^{* *}$

\begin{tabular}{|c|c|}
\hline $\begin{array}{l}\text { Información del } \\
\text { artículo }\end{array}$ & Resumen \\
\hline Recibido: & \\
\hline 1 ener & \multirow{4}{*}{$\begin{array}{l}\text { Este documento analiza los determinantes de la } \\
\text { desigualdad salarial en la manufactura en México. Entre } \\
\text { los determinantes consensuados por la literatura } \\
\text { económica se encuentran la apertura comercial, el cambio } \\
\text { técnico, la oferta de empleo y aspectos institucionales del } \\
\text { mercado de trabajo. A partir de un modelo con datos de } \\
\text { panel, se busca probar la significancia estadística de estas } \\
\text { variables en la determinación de la desigualdad salarial } \\
\text { para los } 21 \text { subsectores manufactureros en México, en el } \\
\text { periodo } 2007-2018 \text {; para ello, se estima el índice de } \\
\text { desigualdad para cada subsector. Los resultados muestran } \\
\text { que la apertura comercial, el cambio técnico, la oferta de } \\
\text { empleo y la reforma laboral inciden de manera directa } \\
\text { sobre la desigualdad salarial. }\end{array}$} \\
\hline $\begin{array}{l}\text { Aceptado: } \\
25 \text { enero } 2021\end{array}$ & \\
\hline $\begin{array}{l}\text { Clasificación JEL: } \\
\text { J31; L60 }\end{array}$ & \\
\hline $\begin{array}{l}\text { Palabras clave: } \\
\text { Desigualdad; Salarios; } \\
\text { Manufactura }\end{array}$ & \\
\hline
\end{tabular}

* Universidad Autónoma del Estado de México, bmv_17_5@hotmail.com; ${ }^{* *}$ Centro de Investigación en Ciencias Económicas de la Facultad de Economía de la Universidad Autónoma del Estado de México.

ISSN Electrónico: 2448-8402 | ISSN Impreso: 1870-221X | (O2021 Los autores @] (1)

Citar asi: Apellido, Murillo-Villanueva, B., Carbajal Suárez, Y., y Almonte, L. (2019). Desigualdad salarial en los subsectores manufactureros en México, 2007-2018. Ensayos Revista de Economía, 40(1), 29-54, DOI:10.29105/ensayos40.1-2 


\begin{tabular}{|c|c|}
\hline Article information & Abstract \\
\hline Received: & \multirow{9}{*}{$\begin{array}{l}\text { This paper analyzes the determinants of wage inequality in } \\
\text { Mexico's manufacture. Among the consensual } \\
\text { determinants in the economic literature are trade openness, } \\
\text { technical change, labor supply, and institutional aspects of } \\
\text { the labor market. Therefore, by means of a panel data } \\
\text { model, this work seeks to prove the statistical significance } \\
\text { of these variables as determinants of wage inequality in the } \\
21 \text { Mexican manufacturing subsectors in the period } 2007- \\
2018 \text {. The results show that trade openness, technical } \\
\text { change, labor supply, and labor reforms impinge positively } \\
\text { on wage inequality. }\end{array}$} \\
\hline 2 January 2020 & \\
\hline Accepted: & \\
\hline 25 January 2021 & \\
\hline JEL Classification: & \\
\hline J31; L60 & \\
\hline Keywords: & \\
\hline Inequality; Wages; & \\
\hline Manufacturing & \\
\hline
\end{tabular}

\section{Introducción}

En los últimos años han tomado relevancia los estudios de la desigualdad salarial, la razón se encuentra en que las cifras de crecimiento económico, aunque son de gran utilidad para analizar el desempeño de largo plazo de una economía, por su carácter agregado, no es un buen indicador para medir la distribución del ingreso y por tanto, las mejoras en el bienestar.

En el contexto internacional, se observa que el crecimiento de la desigualdad es una constante en los países desarrollados y en desarrollo (PNUD, 2010 y OIT, 2012), lo que ocasiona que las mejoras en el bienestar se traduzcan solo para una fracción de la población. También se ha encontrado que la desigualdad del ingreso en países en desarrollo obedece a distintas variables como género, escolaridad, calidad del empleo, ubicación geográfica, sector de actividad económica, entre otros (Ramos et al., 2018). Esta gama de posibilidades sugiere que el análisis de esta variable además de ser multidimensional, es muy amplio. Por otro lado, la desigualdad del ingreso en el nivel nacional se desagrega y es la consecuencia de la desigualdad en distintos niveles y sectores de ocupación. De ahí la necesidad de acotar, cada vez más, el análisis de este problema.

Este trabajo considera que es relevante analizar la desigualdad del ingreso en el sector manufacturero, dado el papel de sector estratégico que juega para la actividad económica (véase Loría et al., 2019; De Jesús, 2019 y Ocegueda, 2003), además de que congrega a las industrias más dinámicas, con mayor progreso tecnológico, científico y de innovación (Sánchez-Juárez y MorenoBrid, 2016). Aunado a esto, la capacidad que posee para generar encadenamientos productivos más allá del propio sector, la generación de empleo formal y calificado, su vinculación con el sector externo y los altos montos de inversión extranjera directa que recibe, caracterizan a este sector como clave para la economía mexicana. Al respecto, Fuentes y García (2009), 
a partir de la aplicación de la teoría de grafos y usando la matriz de insumo producto de 2003, presentan evidencia de la importancia que tiene la industria manufacturera en la estructura productiva de México, por ser fuerte demandante de insumos intersectoriales y oferente de productos intermedios. A decir de los autores, "paso obligado de los flujos sectoriales de la economía" (Fuentes y García, 2009: 150).

En México, entre 2000 y 2018, este sector mostró una participación promedio anual de $17 \%$ en la producción nacional y de $25 \%$ en el empleo nacional (INEGI, 2019). Los datos censales de 2013 señalan que, en ese año, el sector manufacturero participó con $48.2 \%$ de la producción bruta total, con $29 \%$ del valor agregado bruto y con $33.9 \%$ de las remuneraciones totales (INEGI, 2014). De 1999 a 2018, la manufactura recibió 259,526.1 millones de dólares por concepto de inversión extranjera directa (IED), cifra que representó el 49\% del total recibido durante el periodo (Secretaría de Economía, 2019). Lo anterior permite plantear la interrogante sobre si, incluso en las industrias más dinámicas de la economía mexicana, es posible encontrar desigualdad del ingreso.

De acuerdo con la Encuesta Mensual de la Industria Manufacturera (EMIM, 2019), los trabajadores se pueden clasificar según el tipo de trabajo en empleados y obreros. Los primeros se definen como los trabajadores que desempeñan labores de oficina, administración, ventas, contabilidad, labores ejecutivas de planeación, organización, dirección y control. Y los segundos desempeñan trabajos ligados a la operación de maquinaria en la fabricación de bienes, tareas auxiliares y otras actividades propias del proceso productivo (EMIM, 2019). Por su trabajo, los empleados reciben un sueldo; mientras que los obreros, reciben un salario.

Entre 2007 y 2018, 80\% de los trabajadores del sector manufacturero fueron obreros (EMIM, 2019), por ello, gran parte de las remuneraciones totales se destinaron al pago de los salarios, de ahí que en este trabajo se plantee analizar la distribución del ingreso de los obreros en los 21 subsectores, es decir, la desigualdad salarial en los 21 subsectores. Más aún, resulta evidente que el crecimiento de los subsectores de la manufactura ha sido muy heterogéneo, algunos han crecido de manera sostenida y a altas tasas, mientras que otros lo han hecho de forma moderada.

Por ejemplo, la industria alimentaria (311), la de fabricación de equipo de transporte (336) y otras industrias manufactureras (339) son las que más han crecido con tasas superiores al $3 \%$, tanto en la producción como el empleo entre 1988 y 2013; en el mismo periodo, la fabricación de insumos textiles (313) y productos derivados del petróleo (324) han decrecido en términos de producción y empleo (ver Carbajal y Carrillo, 2016). Sin lugar a duda, esto 
también es consecuencia de la relación que cada subsector guarda con variables relevantes, como la apertura comercial, el cambio técnico, entre otros.

La basta literatura nacional e internacional sobre desigualdad salarial sugiere que las condiciones de género, la experiencia (Arceo-Gómez y CamposVázquez, 2014; Contreras y Gallegos, 2007), la flexibilidad laboral (Hernández, 2012), la apertura comercial (Esquivel y Rodríguez, 2003; Rodríguez, Germán-Soto y González, 2017), el comercio (Chiquiar, et al., 2017, Akerman, et al., 2013), el cambio técnico (Acemoglu, 2002, Card y DiNardo, 2002), la calidad del empleo (Zamudio, 2001, Cañonero y Werner, 2002) y la oferta laboral (Topel, 1994, Hernández-Laos, 2000, De la Garza et al., 2001) son algunas variables que explican la desigualdad salarial en el nivel nacional y en la industria. También se encuentran documentos que sugieren el estudio y tratamiento de la desigualdad salarial desde el enfoque de la polarización (García, Fuentes y Montes, 2012; Huesca, 2003).

En este trabajo, el objetivo está centrado en identificar, a partir de indicadores tradicionales de concentración como el índice de Gini, el nivel de desigualdad salarial que prevalece en los 21 subsectores que conforman al sector manufacturero en México, e identificar sus determinantes durante el periodo 2007-2018.

En ese sentido, la hipótesis central de este trabajo es que a diferencia de la desigualdad salarial que prevalece en el nivel nacional, entre 2007 y 2018, se observa baja desigualdad salarial entre los subsectores de la industria manufacturera, que se explica en gran medida por el comercio internacional, el cambio técnico y la flexibilidad laboral. La metodología que se utilizó para probar la hipótesis consiste en obtener el coeficiente de Gini para la industria manufacturera desagregada en subsectores; asimismo, se estima un modelo con datos de panel para medir la significancia estadística de las variables propuestas, como determinantes de la desigualdad salarial, en el nivel de subsector.

El trabajo se desarrolla en cuatro apartados, además de esta introducción y las conclusiones. En el primero, se revisan las relaciones teóricas y empíricas observadas entre los determinantes de la desigualdad salarial. En el segundo, se discuten algunos datos sobre el salario en los subsectores manufactureros en México. En el tercero, se presenta la metodología utilizada para medir la desigualdad salarial y la relación entre variables. En el cuarto, se muestran los resultados sobre la desigualdad salarial entre los subsectores manufactureros y el grado explicativo del comercio internacional, el cambio técnico y la flexibilidad laboral sobre la desigualdad salarial.

\section{Desigualdad salarial y su relación con otras variables}


Como se mencionó, las causas que explican el aumento en la dispersión salarial son diversas, pero se pueden resumir en cuatro aspectos (Castro y Huesca, 2007): i) la apertura comercial, ii) el cambio técnico, iii) cambios en el mercado laboral y $i v$ ) cambios en la oferta de empleo. A continuación, se analiza la forma en que cada uno de estos aspectos influye sobre la desigualdad salarial.

\subsection{Apertura comercial}

De acuerdo con el teorema Stolper-Samuelson (SS) (1941), el comercio afecta al precio relativo de los factores porque ocasiona cambios en el tipo de trabajo que se demanda. De manera clara, el teorema SS indica que si una economía en desarrollo (intensiva en trabajo poco calificado), incrementa su comercio con países desarrollados (intensivos en trabajo calificado), la economía en desarrollo se especializará en la exportación de bienes intensivos en trabajo poco calificado, ocasionado que la demanda y el salario de los trabajadores poco calificados incremente, y que el de los trabajadores calificados, disminuya.

Lo contrario se observará en la economía en desarrollo, esta se especializará en la exportación de bienes intensivos en trabajo calificado, ocasionando que la demanda de este tipo de trabajo aumente, lo mismo que su salario. Por tanto, economías en desarrollo como la mexicana, de mayor comercio con economías desarrolladas, ocasionaría un incremento en la demanda relativa del trabajo no calificado, que se traduce en un incremento en la remuneración al trabajo no calificado, y en un incremento en el salario relativo de este tipo de trabajo, que podría significar una reducción en la desigualdad salarial porque el salario de los trabajadores no calificados experimenta un incremento, como consecuencia del comercio (ver Esquivel y Rodríguez, 2003 y Hoekman y Winters, 2005).

Además, si se considera que en los últimos 10 años, el 88.8\% del comercio mexicano se realiza con países desarrollados como EE. UU., Canadá, Europa y Japón (Banxico, 2019), que se caracterizan por ser países abundantes en trabajo calificado, entonces México debió experimentar una reducción en la desigualdad salarial como resultado del comercio.

De acuerdo con Chiquiar et al. (2017), la integración de México con países desarrollados como EE. UU. y Canadá a través del TLCAN, ahora T-MEC, llevó a que el empleo manufacturero de trabajadores no calificados aumentara $\mathrm{y}$ a que sus salarios reales fueran mayores. Estos resultados coinciden con los que reportan Cañonero y Werner (2002) y Esquivel y Rodríguez (2003) quienes argumentan que con la inserción de México al GATT la desigualdad salarial se incrementó como resultado del aumento en la oferta de empleo no calificado, pero que se redujo después de la instrumentación del TLCAN 
porque aumentó el comercio con países abundantes en trabajo calificado. Estos hallazgos apoyan el cumplimiento del teorema SS en México.

Sin embargo, se ha encontrado que el comercio y las reformas al comercio explican solo una pequeña fracción del cambio en la desigualdad salarial, observado en países desarrollados y en desarrollo. De acuerdo con Hoekman y Winters (2005: 24), “... las magnitudes de los efectos de mayor comercio en países de la OECD sobre los salarios y la desigualdad son pequeñas."

Por otro lado, en el trabajo de Chiquiar et al. (2017), hay evidencia que sugiere que el comercio tiene efectos diferenciados en las regiones de México; y, que las ciudades del norte, al tener mayor exposición al TLCAN, han experimentado mayor crecimiento en los salarios. Además, Akerman, et al. (2013), argumentan que la apertura comercial puede incrementar la desigualdad salarial al interior de la industria manufacturera y de los subsectores, ya que no todas las empresas exportan y no todas se benefician del consecuente incremento salarial. Harrison y Hanson (1999) refieren que al interior de las industrias, la desigualdad salarial es mayor cuanto mayor es su presencia en actividades de exportación.

\subsection{Cambio técnico}

El cambio técnico se define como el cambio en la estructura y contenido de la producción, e implica un mejoramiento o un cambio en las dinámicas de producción existentes, en el conjunto de factores que se combinan, o en las cantidades que se utilizan. Murillo-Villanueva (2019) da evidencia de que entre 2003 y 2012 el cambio técnico en los subsectores manufactureros de México fue gradual, y con cambios importantes en la intensidad con la que se utilizan los insumos primarios. Particularmente, se observa que la mayoría de los subsectores experimentan una reducción en el empleo, que sugiere una relación sustitutiva entre capital y trabajo de baja calificación, y una relación de complementariedad entre capital y trabajo calificado.

El cambio técnico ocasiona variaciones en la composición del empleo a favor del trabajo calificado e incrementa la desigualdad salarial. De acuerdo con Broecke (2016), la globalización, los cambios en la demanda de capacidades y los cambios organizacionales han incrementado el pago a las capacidades y, por tanto, la desigualdad salarial.

Esquivel y Rodríguez (2003) encuentran que en la industria manufacturera el cambio técnico llevó a una reducción en los salarios reales de los trabajadores no calificados, y a un incremento en el salario real de los calificados, presionando a la alza la desigualdad entre calificados y no calificados entre 1988 y 2000. Al respecto, hay diversos trabajos que indican que la calificación 
del trabajo influye sobre los salarios, y que los trabajadores con mayor calificación tienen en promedio mayores salarios que los no calificados (Krugman y Lawrence, 1993; Hoekman y Winters, 2005 y Cañonero y Werner, 2002).

En algunos de estos trabajos se ha utilizado a la variable productividad laboral como proxy del cambio técnico. La idea detrás de este supuesto es que el cambio técnico incrementa la productividad laboral, vía la reducción en el uso de trabajo. Castellano (2010) presenta evidencia de que, en la industria manufacturera, el incremento de la productividad laboral se traduce en un incremento en los salarios. Los diferenciales entre las productividades laborales entre subsectores podrían llevar a distintos niveles de salario, y a agravar la desigualdad salarial.

También se considera que el perfil tecnológico de los subsectores manufactureros es un indicador de cambio técnico, que contribuye a la explicación de la desigualdad salarial en el sentido de que, a mayor innovación, la desigualdad se agudiza. Se utiliza la clasificación de la Organización para la Cooperación y el Desarrollo (OCDE), basada en indicadores de intensidad tecnológica que reportan Carbajal-Suárez, Mejía-Reyes y Rendón (2008) y que clasifica a los subsectores de la manufactura en cuatro categorías: alta tecnología, tecnología alta-media, tecnología baja-media y tecnología baja.

\subsection{Cambios en el mercado laboral}

La fragmentación de los procesos productivos de las grandes empresas y la creciente relevancia de las cadenas globales de valor han tenido como principal objetivo la búsqueda de bajos costos laborales y el incremento en la tasa de ganancia. Por ello se han desarrollado nuevas formas de organización del trabajo que, de acuerdo con Hernández (2012: 34), "no siempre resultan benéficas para los trabajadores".

La nueva organización del trabajo es resultado de las reformas laborales que fomentan la flexibilidad laboral y la reducción de la participación sindical como vías para incentivar el empleo. En la actualidad, se observan contrataciones sin seguridad laboral y con remuneración flexible determinada por la duración de la jornada laboral, el rendimiento, la productividad del trabajo o los beneficios de la empresa (ver Neffa, 2010). Hernández (2012) refiere que, en 2011 , el $56.88 \%$ de los trabajadores mexicanos tuvieron empleos temporales, sin contrato formal o con contratos que no especificaban la duración. En ese sentido, la reforma laboral de 2012 que tuvo por objetivo fomentar el acceso al mercado laboral a través de la flexibilidad laboral, podría traducirse en un incremento en la desigualdad salarial. 
Aunado a esto, la reducción de la participación sindical también afecta a la desigualdad salarial, especialmente, reduce las remuneraciones de los trabajadores menos calificados. No obstante, de acuerdo con Cragg y Epelbaum (1996), Bell (1997) y López-Acevedo (2001), hasta finales del S. $\mathrm{XX}$, en México, la pérdida de poder sindical no afectó la evolución de la desigualdad salarial.

\subsection{Cambios en la oferta de empleo}

Respecto a la oferta de empleo, se ha encontrado evidencia que sugiere que la participación femenina y juvenil, así como el desempleo, son variables que afectan la oferta relativa de empleo y que inciden sobre la desigualdad salarial. Topel (1994) sugiere que la oferta laboral femenina afecta el salario de los hombres menos calificados. En el mismo sentido, De la Garza et al. (2001), encuentran que en México las mujeres se emplean en actividades de baja productividad ocasionando que la brecha salarial sea más amplia.

Contrario a la idea de que la liberalización comercial ha intensificado la desigualdad salarial entre hombres y mujeres, Aguayo-Tellez, Airola y Juhn (2010) dan evidencia de que la liberalización comercial en México, aumentó la participación de las mujeres en el mercado laboral manteniendo estable la relación salarial entre hombres y mujeres.

Por otro lado, Meza (2005) encuentra que la participación de los jóvenes aumenta la desigualdad salarial al incrementar la oferta de empleo y abaratar los salarios. La presión que en México ejercen los jóvenes sobre la desigualdad salarial también podría ser relevante, si se considera que cada año se incorporan al mercado laboral más de un millón de jóvenes (ver HernándezLaos, 2000).

Además, de acuerdo con los resultados que reporta Castellano (2010) para México, en el corto plazo, un incremento de $1 \%$ en la tasa de desempleo de la industria manufacturera, reduce el incremento del salario nominal del sector en $0.54 \%$. Lo anterior sugiere que el incremento en la oferta laboral detiene el crecimiento de los salarios y como consecuencia, incrementa la desigualdad salarial.

\section{Salario en los subsectores manufactureros}

De acuerdo con el Sistema de Clasificación Industrial de América del Norte (SCIAN, 2013), el sector manufacturero se puede desagregar en 21 subsectores. El nivel de calificación y las habilidades requeridas en los trabajadores diferirá entre industrias, debido a las diferencias de productos, 
procesos de producción y contenido tecnológico, lo que se reflejará en los salarios.

En la tabla 1, se presenta el salario real unitario de los obreros que se emplean en los distintos subsectores manufacturero en 2007 y 2018. En ambos años, los datos muestran una notable heterogeneidad salarial entre subsectores. En 2007 el subsector fabricación de productos textiles (314) registró un salario medio real unitario de 31 pesos por hora, el más bajo de la industria manufacturera; mientras que en la industria de derivados del petróleo (324), el salario medio fue de 115 pesos por hora, el mayor en el sector manufacturero y casi cuatro veces mayor que en el primero.

En cambio, en 2018, el menor salario unitario fue de 34 pesos por hora y se registró en la industria de la madera, el mayor salario unitario fue de 98 pesos por hora y nuevamente se registró en la industria de derivados del petróleo (324). En 2007, los salarios de la manufactura oscilaron entre los 31 y 115 pesos por hora, mientras que en 2018 el rango fue de 34 a 98 pesos por hora; esto indica que para 2018, la brecha entre el menor y el mayor salario real unitario disminuyó, lo que podría sugerir una reducción en la desigualdad salarial de la manufactura en los últimos años, como consecuencia de una reducción en el salario de los subsectores con el salario más alto, y del aumento en salario de los subsectores con el salario más bajo.

Finalmente, es importante mencionar que la disparidad en los salarios puede obedecer a las diferencias en calificación, capacidades y habilidades de la mano de obra en las distintas industrias. Se puede observar que los subsectores basados en recursos naturales y de bajo nivel tecnológico, como la industria alimentaria (311), bebidas y tabaco (312), insumos textiles (313), productos textiles (314), prendas de vestir (315), productos de cuero y piel (316), industria de la madera (321), del papel (322), impresión y conexas (323), muebles, colchones, etc. (327), y otras manufacturas (339), ofrecen los salarios más bajos del sector manufacturero, lo que se podría explicar por una menor calificación de la mano de obra. 
Tabla 1

Salario medio real unitario en los subsectores manufactureros, 2007 y 2018 (pesos de 2013 por hora)

\begin{tabular}{|c|c|c|c|}
\hline \multicolumn{2}{|l|}{2007} & \multicolumn{2}{|l|}{2018} \\
\hline Salario & SCIAN/Subsector & Salario & SCIAN/Subsector \\
\hline$\$ 31$ & 314 Productos textiles & $\$ 34$ & 321 Industria de la madera \\
\hline$\$ 32-45$ & $\begin{array}{ll}311 & \text { Alimentaria } \\
312 & \text { Bebidas y tabaco } \\
313 & \text { Insumos textiles } \\
315 & \text { Prendas de vestir } \\
316 & \text { Productos de cuero y } \\
\text { piel } & \\
321 & \text { Industria de la madera } \\
322 & \text { Industria del papel } \\
323 & \text { Impresión y conexas } \\
337 & \text { Muebles, colchones, } \\
\text { etc. } & \\
339 & \text { Otras manufacturas } \\
\end{array}$ & $\$ 35-45$ & $\begin{array}{ll}311 & \text { Alimentaria } \\
312 & \text { Bebidas y tabaco } \\
313 & \text { Insumos textiles } \\
314 & \text { Productos textiles } \\
315 & \text { Prendas de vestir } \\
316 & \text { Productos de cuero y } \\
\text { piel } & \\
334 & \text { Cómputo y } \\
\text { comunicación } \\
337 \text { Muebles, colchones, } \\
\text { etc. } \\
339 \text { Otras manufacturas } \\
\end{array}$ \\
\hline$\$ 46-56$ & $\begin{array}{l}326 \text { Ind. del plástico y } \\
\text { hule } \\
327 \text { Productos a base de } \\
\text { minerales no metálicos } \\
331 \text { Metálicas básicas } \\
332 \text { Productos metálicos } \\
333 \text { Maquinaria y equipo } \\
334 \text { Cómputo y } \\
\text { comunicación } \\
335 \text { Accesorios y ap. } \\
\text { eléctricos } \\
336 \text { Eq. de transporte }\end{array}$ & $\$ 46-56$ & $\begin{array}{l}322 \text { Industria del papel } \\
323 \text { Impresión y conexas } \\
326 \text { Ind. del plástico y } \\
\text { hule } \\
327 \text { Productos a base de } \\
\text { minerales no metálicos } \\
332 \text { Productos metálicos } \\
333 \text { Maquinaria y equipo } \\
335 \text { Accesorios y ap. } \\
\text { eléctricos }\end{array}$ \\
\hline$\$ 70$ & 325 Química & $\$ 61-68$ & $\begin{array}{ll}325 & \text { Química } \\
331 & \text { Metálicas básicas } \\
336 & \text { Eq. de transporte }\end{array}$ \\
\hline$\$ 115$ & $\begin{array}{l}324 \text { Derivados del } \\
\text { petróleo }\end{array}$ & $\$ 98$ & $\begin{array}{l}324 \text { Derivados del } \\
\text { petróleo }\end{array}$ \\
\hline
\end{tabular}

Fuente: elaborado con datos de la EMIM (INEGI, 2019).

\section{Metodología y datos}

\subsection{Desigualdad salarial}

En este trabajo, la desigualdad salarial se refiere a la desigualdad en la distribución de los salarios entre obreros que se emplean en el mismo subsector, pero en distintas ramas. Para su medición, se utilizaron los datos de salarios reales por hora en cada una de las 86 ramas manufactureras que conforman los 21 subsectores y que reporta la Encuesta Mensual de la Industria Manufacturera (EMIM, 2019), para el periodo 2007-2018; algunos subsectores 
se desagregan en 9 ramas, mientras que otros solo en 2. A partir de esta información, se calculó la desigualdad salarial al interior de los subsectores y entre ramas.

Para la obtención de medidas representativas de la desigualdad, se han utilizado distintos índices derivados de la curva de Lorenz (1905) como el coeficiente de Gini (1914), el índice de Theil (1 y 2), el índice de diferencia absoluta media (AGC); también se han utilizado distintos indicadores de dispersión como los índices de entropía generalizada (ver Atkinson y Bourguignon, 2000, Cowell, 2000 y Heshmati, 2004).

Aun cuando en la literatura se han encontrado diversas medidas de desigualdad, en este trabajo se utiliza el índice de Gini (1914). Este coeficiente es un instrumento para medir la concentración de ingresos entre la población de un país o región en un periodo determinado; se obtiene del área entre la curva de perfecta igualdad y la curva de Lorenz dividida por el área debajo de la línea de perfecta igualdad, se calcula a partir de la ecuación (1).

$G_{i t}=\frac{\sum_{i=1}^{n-1}\left(p_{i}-q_{i}\right)}{\sum_{i=1}^{n-1} p_{i}}$

Donde $q$ representa la proporción acumulada del producto entre salario unitario $\mathrm{y}$ horas trabajadas, $p$ la proporción acumulada de horas trabajadas por los obreros en las $i$ ramas manufactureras. Recuérdese que el índice de Gini toma valores entre 0 y 1 , donde 0 representa un escenario de perfecta igualdad en la distribución de los salarios (todos tienen el mismo salario), y 1 representa la perfecta desigualdad salarial (una proporción de los trabajadores acapara todos los salarios).

Las ventajas de uso residen en que permite comparar las distribuciones de ingresos entre los subsectores manufactureros, es de fácil interpretación, se puede usar para indicar cómo ha cambiado la distribución del ingreso en los subsectores en el tiempo, además de que satisface las propiedades deseables que deben cumplir las medidas de desigualdad: invariancia a la escala, invariancia a las réplicas, simetría y el principio de transferencias, (o condición de Dalton-Pigou) (véase Atuesta, Mancero y Tromben, 2018). Este último establece que las transferencias de ingresos de trabajadores con altos salarios, a los de bajos salarios resultan en una distribución más equitativa.

Como se observa más adelante, el indicador de brecha salarial $B S_{i t}$ también arroja resultados significativos en el análisis de los determinantes de la desigualdad salarial en los subsectores manufactureros. Este indicador se construyó para cada subsector, a partir de la diferencia salarial entre la rama manufacturera con el salario más alto y la rama con el salario más bajo. 


\subsection{Determinantes de la desigualdad salarial}

Una vez obtenido el indicador de la desigualdad salarial, se plantea un modelo con datos de panel para la industria manufacturera que busca probar la significancia estadística de algunas de las variables revisadas en el primer apartado, como determinantes de la desigualdad salarial.

Para el caso que nos ocupa, se utilizan las variables siguientes para explicar la desigualdad salarial medida a través del coeficiente de Gini $\left(G_{i t}\right)$ y de la brecha salarial $\left(B S_{i t}\right)$ : el volumen de comercio exterior del subsector $i$ en el periodo $t$ $\left(C_{i t}\right)$, que se define como la suma de las exportaciones e importaciones y que mide la intensidad de la apertura comercial; el índice de productividad laboral relativa ${ }^{1}$ del subsector $i$ en el periodo $t\left(P_{i t}\right)$, el índice de subcontratación ${ }^{2}$ del subsector $i$ en el periodo $t\left(S_{i t}\right)$, el total de obreros que se emplean en el subsector $i$ y periodo $t\left(L_{i t}\right)$, y el perfil tecnológico del subsector i en el periodo $\mathrm{t}\left(P T_{i t}\right)$. En el anexo 1, se presentan las estadísticas básicas de estas variables.

Por la evidencia de trabajos previos, que consideran a la calificación laboral como un factor central de la desigualdad salarial, quizá sea discutible que en este trabajo se considere la desigualdad entre obreros. Sin embargo, el interés por considerar este tipo de trabajadores se debe a que parte importante de la estructura del empleo, en el sector manufacturero, se compone de obreros; cifra que entre 2007 y 2018 ascendió a $80 \%$. En este caso, el volumen de comercio se utiliza como un indicador absoluto de apertura comercial, el índice de productividad laboral y el perfil tecnológico como variables proxy del cambio técnico, y el índice de subcontratación como variable de flexibilidad laboral. El indicador de flexibilidad laboral trata de incorporar los efectos que ha tenido la reforma laboral en México, a partir de su instrumentación formal en 2012, y de que entre los principales cambios a la Ley Federal del Trabajo, se plantea la regulación del outsourcing, contratos temporales a prueba y pagos por jornada laboral reducida, bajo el supuesto que estos mecanismos tendrían la capacidad de insertar a jóvenes y mujeres al mercado laboral (véase Loría, Ramírez y Salas, 2015).

$1 P_{i}=\left[\left(\frac{P I B_{i}}{H_{i}}\right) /\left(\frac{P I B_{n}}{H_{n}}\right)\right]$, donde el numerador expresa el producto por hora-trabajo de obrero en el subsector de actividad $i$, y el denominador el producto por hora-trabajo de obrero en el total de la industria manufacturera $n$.

$2 S_{i}=\left[H S_{i} /_{H_{i}}\right]$ donde el numerador es el número de horas trabajadas por obreros suministrados por otra razón social en el subsector $i$, y el denominador es el total de horas obrero en el mismo subsector $i$. 
Con relación al tema, Loría, Ramírez y Salas (2015) proponen, para la realidad del mercado de trabajo en México, utilizar un indicador de flexibilidad laboral factual, que comprenda la tasa porcentual de trabajadores eventuales al total de trabajadores en el sector formal, siendo este un indicador de flexibilidad laboral numérica que representa la facilidad de contratación y despido en el mercado formal de trabajo en México. De ahí que el índice de subcontratación, en este trabajo, se considera como un indicador de flexibilidad laboral y por último, al nivel de empleo como variable de cambios en el mercado laboral.

Los datos de comercio total por subsector se obtuvieron del BIE (INEGI, 2019), mientras que los índices de productividad laboral y subcontratación se construyeron con información de la Encuesta Mensual de la Industria Manufacturera (EMIM, 2019). El modelo de datos de panel se estimó a partir de la ecuación (2).

$$
\begin{array}{r}
G_{i t}=\alpha+\alpha_{1} C_{i t}+\alpha_{2} P_{i t}+\alpha_{3} S_{i t}+\alpha_{4} L_{i t}+\alpha_{5} P T_{i t}+u_{i t} \\
i=1, \ldots, N ; \quad t=1, \ldots, T
\end{array}
$$

Donde: $i$ se refiere a la unidad de estudio de corte transversal (subsectores manufactureros) y $t$ a la dimensión en el tiempo (datos anuales), la muestra total del modelo sería $\mathrm{N}=\mathrm{nxT}$, donde $\mathrm{n}$ corresponde a 21 , que son los subsectores de la manufactura y $\mathrm{T}$ es igual a 12 , que son los años que corresponden al periodo 2007-2018. Se tiene un panel balanceado con $\mathrm{N}$ igual a 252 observaciones. Los parámetros $\alpha, \alpha_{1}, \alpha_{2}, \alpha_{3}, \alpha_{4} y \alpha_{5}$ son los que estima el modelo, y el término $u_{i t}$ hace referencia a los errores del modelo. En un modelo con datos de panel, el efecto de un cambio en las variables explicativas es el mismo para las $i$ unidades de estudio en los $t$ periodos, y la heterogeneidad se considera en el término de error $u_{i t}$ (Wooldridge, 2002). Esta última puede variar entre unidades de estudio $i$ o entre unidades $i$ y tiempo $t$ (Hsiao, 2003). En ese sentido, el término de error $u_{i t}$ se compone de un efecto individual $\left(m_{i}\right)$ $\mathrm{y}$ de un término puramente aleatorio $\left(e_{i t}\right)$, de manera que la ecuación (2) se puede reescribir de la siguiente forma.

$G_{i t}=\alpha+\alpha_{1} C_{i t}+\alpha_{2} P_{i t}+\alpha_{3} S_{i t}+\alpha_{4} L_{i t}+\alpha_{5} P T_{i t}+m_{i}+e_{i t}$

El componente de efecto individual puede tomar distintos valores según la heterogeneidad entre unidades de estudio. Si $m_{i}=0$, se dice que no existe heteogéneidad no observable entre individuos, por lo que, tanto la ordenada al origen como las variables explicativas tienen el mismo efecto sobre todos los subsectores. En este caso, la ecuación del modelo sería como en la ecuación (3a) y representa un modelo de regresión agrupada o pooled.

$G_{i t}=\alpha+\alpha_{1} C_{i t}+\alpha_{2} P_{i t}+\alpha_{3} S_{i t}+\alpha_{4} L_{i t}+\alpha_{5} P T_{i t}+e_{i t}$ 
Si $m_{i} \neq 0$, distinto para cada unidad de estudio $i$ y constante a lo largo de $T$, se concluye que las variables explicativas tienen el mismo efecto sobre todas las unidades, pero que la ordenada al origen $\alpha$ es diferente para cada unidad y viene dado por $m_{i}$. Este modelo se representa con la ecuación ( $3 b$ ) y se conoce como modelo de efectos fijos.

$$
G_{i t}=m_{i}+\alpha_{1} C_{i t}+\alpha_{2} P_{i t}+\alpha_{3} S_{i t}+\alpha_{4} L_{i t}+\alpha_{5} P T_{i t}+e_{i t}
$$

Por último, si $m_{i} \neq 0$ y toma valores distintos para cada unidad y a lo largo del periodo, se dice que $m_{i}$ es una variable aleatoria. En este caso, los efectos individuales se distribuyen aleatoriamente alrededor de cierto valor; este modelo incorpora la heterogeneidad no observable en el término de error y no en la constante. A este tipo de modelos se le conoce como de efectos aleatorios y se representa con la ecuación (3), donde $m_{i}+e_{i t}=u_{i t}$.

\section{Estimación y discusión de resultados}

\subsection{Desigualdad salarial entre los subsectores manufactureros}

En un primer ejercicio, se estimó el coeficiente de Gini para medir la desigualdad salarial entre los subsectores de la industria manufacturera (ver gráfica 1). La gráfica 1 indica que la desigualdad salarial en la industria manufacturera es relativamente baja, oscila entre 0.13 y 0.17 , se incrementó en la primera mitad del periodo como consecuencia de la Gran Recesión, pero comenzó a reducirse a partir del 2012. La reducción en la desigualdad salarial, en la segunda mitad del periodo, se explica por el efecto transitorio de la Gran Recesión en los salarios y, por una fuerte caída en el salario real de los subsectores de mayor salario, acompañado de un ligero incremento en el salario real de los subsectores con menor salario (ver tabla 1).

Los resultados se presentan en dos grupos. Por un lado, los subsectores de menor desigualdad salarial y, por otro lado, los de mayor desigualdad. Del total de 21 subsectores, 15 se encuentran en la primera categoría (ver tabla 2), y 6 en la segunda (ver gráfica 2). 


\section{Gráfica 1}

Desigualdad salarial entre subsectores de la industria manufacturera, 2007-2018

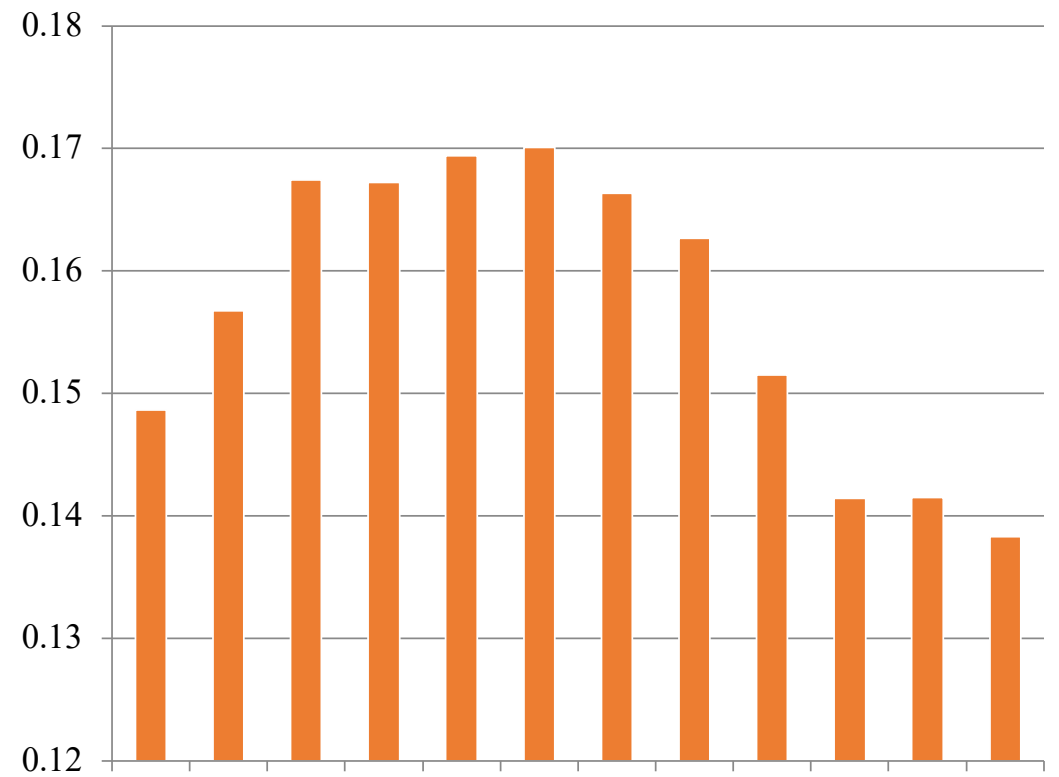

200720082009201020112012201320142015201620172018

Fuente: estimaciones realizadas con datos de la EMIM (INEGI, 2019).

Ampliando el nivel de desagregación de las actividades manufactureras, se estimó la desigualdad salarial al interior de los subsectores manufactureros, es decir, entre las ramas de cada subsector.

En la tabla 2, se muestran los subsectores del primer grupo. Los resultados muestran que la desigualdad salarial en estos subsectores es muy baja, en la mayoría de los casos y años, el índice no rebasa la cifra de 0.1 ; además, la mayoría de estos subsectores se caracteriza por registrar los niveles más bajos de salario real por hora. Esto indica que la desigualdad salarial de los subsectores de textil y de calzado $(313,314,315$ y 316$)$, de la industria de la madera (321), del papel (322), plástico (326), minerales no metálicos (327), productos metálicos (332), maquinaria y equipo (333), computación y comunicación (334), aparatos eléctricos (335), muebles (335) y otras manufacturas (339) es baja, porque los trabajadores reciben salarios que son bajos y que son muy similares entre ramas. 
Tabla 2

Desigualdad salarial en los subsectores manufactureros con el menor índice de Gini, 2007-2018

\begin{tabular}{lllllll}
\hline SCIAN & Descripción & $\mathbf{2 0 0 7}$ & $\mathbf{2 0 1 0}$ & $\mathbf{2 0 1 3}$ & $\mathbf{2 0 1 5}$ & $\mathbf{2 0 1 8}$ \\
\hline 313 & Insumos textiles & 0.028 & 0.029 & 0.033 & 0.051 & 0.032 \\
314 & Productos textiles & 0.015 & 0.008 & 0.036 & 0.033 & 0.030 \\
315 & Prendas de vestir & 0.112 & 0.059 & 0.036 & 0.081 & 0.075 \\
316 & Productos de cuero y piel & 0.045 & 0.039 & 0.030 & 0.046 & 0.048 \\
321 & Industria de la madera & 0.043 & 0.030 & 0.029 & 0.047 & 0.050 \\
322 & Industria del papel & 0.011 & 0.020 & 0.033 & 0.022 & 0.023 \\
323 & Impresión e industrias & 0.013 & 0.029 & 0.007 & 0.026 & 0.018 \\
& conexas & & & & & \\
326 & Industria del plástico y del & 0.078 & 0.088 & 0.099 & 0.070 & 0.021 \\
327 & hule & & & & & \\
332 & Productos a base de & 0.133 & 0.121 & 0.067 & 0.066 & 0.064 \\
333 & Prinerales no metálicos & & & & & \\
334 & Maquinaria y equipo & 0.084 & 0.077 & 0.093 & 0.101 & 0.085 \\
335 & Equipo de computación, & 0.072 & 0.068 & 0.060 & 0.087 & 0.085 \\
337 & comunicación, etc. & & 0.089 & 0.071 & 0.060 & 0.059 \\
339 & Mccesorios y ap. eléctricos & 0.045 & 0.042 & 0.044 & 0.038 & 0.041 \\
\hline
\end{tabular}

Fuente: estimaciones realizadas con datos de la EMIM (INEGI, 2019).

En la gráfica 2, se muestran los resultados del índice de Gini para los subsectores con mayor desigualdad salarial entre 2007 y 2018. En la mayoría de los subsectores, y a lo largo de este periodo, el índice de Gini oscila entre 0.1 y 0.3 .

Vemos que el subsector con la desigualdad salarial más elevada es el de fabricación de derivados del petróleo. Esto indicaría que, en términos de salarios, no es lo mismo trabajar en la rama de refinación de petróleo (324110), que en la de fabricación de productos de asfalto (324120) o en la de fabricación de aceites y grasas (324191); los salarios difieren de manera importante entre las tres ramas del mismo subsector.

La industria química (325) es otro subsector donde la desigualdad salarial es elevada, aunque en los últimos años, ha reducido de manera importante (ver gráfica 2). En los subsectores de la industria alimentaria y de metálicas básicas la desigualdad salarial se ha mantenido relativamente constante. Por el contrario, en la industria de bebidas y tabaco, la desigualdad se ha incrementado. 
La evidencia de que en la mayoría de los subsectores la desigualdad salarial entre ramas de un mismo subsector se ha reducido, explica porqué el índice de Gini total manufacturero se ha reducido en los últimos años.

\section{Gráfica 2}

Desigualdad salarial en los subsectores manufactureros con el mayor índice de Gini, 2007-2018

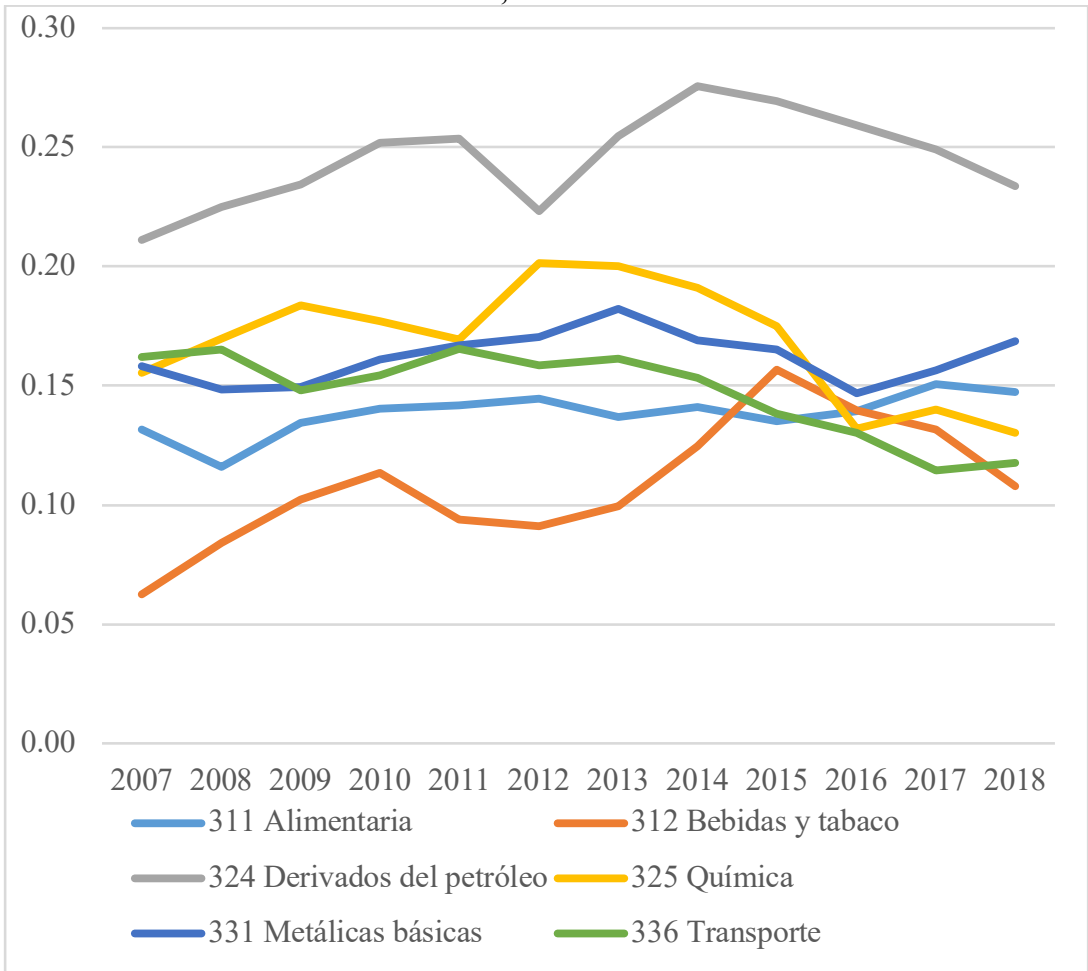

Fuente: estimaciones propias con datos de la EMIM (2019).

\subsection{Determinantes de la desigualdad en los subsectores manufactureros}

A partir de los índices de desigualdad salarial de los 21 subsectores manufactureros, se integró un panel de datos balanceado de 252 observaciones, resultado de 21 unidades transversales, una para cada subsector y 12 unidades temporales que corresponden al periodo 2007-2018. Se estimó la desigualdad salarial de los subsectores de la manufactura a partir de la especificación de las ecuaciones (3a) y (3b), de la siguiente forma:

$\ln G_{i t}=\alpha+\alpha_{1} \ln C_{i t}+\alpha_{2} P_{i t}+\alpha_{3} S_{i t}+\alpha_{4} \ln L_{i t}+\alpha_{5} P T_{i t}+e_{i t}$ 
donde:

$\ln G_{i t}=$ coeficiente de Gini (en logaritmos) de la manufactura, del subsector 1 al 21.

$\ln C_{i t}=$ el volumen de comercio exterior de la manufactura, del subsector 1 al 21 (en logaritmos).

$P_{i t}=$ índice de productividad laboral de la manufactura, del subsector 1 al 21. $S_{i t}=$ índice de subcontratación de la manufactura, del subsector 1 al 21.

$\ln L_{i t}=$ población ocupada en la manufactura, del subsector 1 al 21 (en logaritmos).

$P T_{i t}=$ perfil tecnológico, del subsector 1 al 21.

Con el propósito de aportar mayor evidencia de los determinantes de la desigualdad salarial, se estimó la ecuación (4), considerando a la brecha salarial como variable dependiente.

Se estimaron modelos de datos agrupados, de efectos fijos y de efectos aleatorios y se aplicaron las pruebas de datos agrupados (pool test) y la de Hausman, que contrasta las estimaciones de efectos fijos y efectos aleatorios. Si se encuentran diferencias significativas, se rechaza la hipótesis nula de igualdad debido a que seguiría existiendo correlación entre el término de error y los regresores; en tal caso, se opta por el modelo de efectos fijos. Si, por el contrario, las estimaciones son significativamente iguales, se elige el modelo de efectos aleatorios. Se concluyó que el modelo de datos agrupados es consistente con efectos fijos, por lo que no fue necesario estimar los modelos con efectos aleatorios. Los resultados de las pruebas arrojaron valores del estadístico F con probabilidades mayores a 0.05 (ver tabla 3).

A partir de la evidencia empírica y la revisión de literatura que se presentó en el apartado 1, se esperaba encontrar las siguientes relaciones entre las variables elegidas, como determinantes de la desigualdad salarial: $a$ ) el incremento en el volumen de comercio $\left(\ln C_{i t}\right.$ ) ha incrementado la desigualdad salarial en los subsectores, a causa de la marcada heterogeneidad en términos de su vinculación con el sector externo; b) mayor productividad laboral $\left(P_{i t}\right)$ sugiere cambio técnico, que lleva a un incremento en la desigualdad salarial vía una reducción en los salarios de los trabajadores no calificados, y un incremento en los de los calificados; $c$ ) a mayor subcontratación laboral $\left(S_{i t}\right)$, medida de la flexibilidad laboral, mayor desigualdad salarial; $d$ ) una relación positiva con el crecimiento del empleo $\left(\ln L_{i t}\right)$; e) una relación positiva con el perfil tecnológico $\left(P T_{i t}\right)$, en el sentido de que a mayor perfil tecnológico, se agudiza la desigualdad salarial en los sectores de actividad.

Al estimar la función de desigualdad salarial a partir del índice de Gini, como se expresa en la ecuación 4, destacan los resultados siguientes: el crecimiento 
de la productividad del trabajo $\left(P_{i t}\right)$, de la subcontratación laboral $\left(S_{i t}\right)$ y del empleo $\left(L_{i t}\right)$ aportan evidencia para considerar que son elementos que contribuyen al incremento de la desigualdad salarial en la manufactura.

Por la magnitud de los coeficientes $S_{i t}(0.092), \ln L_{i t}(0.027)$ y $P_{i t}(0.019)$, los resultados indican que las condiciones que prevalecen para la contratación del factor trabajo, en los subsectores de la manufactura asociados a la flexibilidad laboral, han sido una de las principales causas para explicar la desigualdad salarial en los subsectores de la manufactura (ver tabla 3), evidencia que coincide con los trabajos de Neffa (2010) y Hernández (2012). Por su parte, se probó que el aumento en la oferta laboral, conduce a un aumento en la desigualdad salarial tal y como lo sugiere Castellano (2010). Y que el incremento de la productividad laboral (variable proxy del cambio técnico y de la calificación laboral), influye de manera directa en el crecimiento de la desigualdad salarial, como lo recomienda la revisión teórica del apartado 1 (ver Cañonero y Wernes, 2002; Esquivel y Rodríguez, 2003 y Hoekman y Winters, 2005).

Si bien se ha argumentado que el proceso de apertura comercial favorecería el crecimiento de la economía por las bondades del tamaño de mercado, y entre los beneficios se observaría un mejoramiento en los niveles salariales de los trabajadores mexicanos, la evidencia indica que el coeficiente de $\ln C_{i t}$ (0.001 ), variable proxy de la apertura comercial, no es significativa; aunque el signo sugiere que puede ser una condición para disminuir la brecha salarial en los subsectores de la manufactura, como lo indica la revisión teórica. En relación con el perfil tecnológico $P T_{i t}$ que prevalece en los subsectores de la manufactura, el coeficiente estimado no es significativo, sin embargo, por el signo también dejaría ver la posibilidad de que pueda explicar la desigualdad salarial que prevalece en la manufactura.

A partir de estos resultados, se buscó evidencia adicional, por lo que se construyeron indicadores de desigualdad salarial adicionales al índice de Gini. Específicamente un índice de entropía generalizada, un índice de desigualdad intersectorial y el indicador de brecha salarial $\left(B S_{i t}\right)$. Al trabajar con el índice de Theil y con el índice de desigualdad intersectorial no se encontraron resultados consistentes, pero sí con el indicador de brecha salarial $\left(B S_{i t}\right)$. 
Tabla 3

Estimación de la desigualdad salarial por subsector de la manufactura, 20072018

\begin{tabular}{|c|c|c|c|c|}
\hline & \multicolumn{2}{|c|}{$\begin{array}{l}\text { Variable dependiente: índice de } \\
\text { Gini (IG) }\end{array}$} & \multicolumn{2}{|c|}{$\begin{array}{l}\text { Variable dependiente: brecha } \\
\text { salarial }\end{array}$} \\
\hline & $\begin{array}{l}\text { Datos } \\
\text { agrupados }\end{array}$ & Efectos fijos & $\begin{array}{l}\text { Datos } \\
\text { agrupados }\end{array}$ & Efectos fijos \\
\hline constante & $-0.250(0.00)$ & & $-51.62(0.00)$ & \\
\hline$P_{i t}$ & $0.019(0.00)$ & $0.019(0.00)$ & $8.465(0.00)$ & $8.616(0.00)$ \\
\hline$S_{i t}$ & $0.092(0.00)$ & $0.092(0.00)$ & $40.869(0.00)$ & $39.820(0.00)$ \\
\hline $\ln C_{i t}$ & $-0.001(0.54)$ & $-0.001(0.50)$ & $-2.430(0.00)$ & $-3.049(0.00)$ \\
\hline $\ln L_{i t}$ & $0.027(0.00)$ & $0.027(0.00)$ & $7.661(0.00)$ & $7.944(0.00)$ \\
\hline$P T_{i t}$ & $0.005(0.62)$ & $0.006(0.59)$ & $25.369(0.00)$ & $27.287(0.00)$ \\
\hline $\mathrm{R}^{2}$ & 0.76 & 0.76 & 0.81 & 0.81 \\
\hline $\mathrm{R}^{2}$ ajustado & 0.76 & 0.75 & 0.80 & 0.80 \\
\hline F estadístico & $160.9(0.00)$ & $155.7(0.00)$ & $213.42(0.00)$ & $208.031(0.00)$ \\
\hline $\begin{array}{l}\text { Prueba de datos } \\
\text { agrupados }\end{array}$ & $0.263(0.99)$ & & $0.386(0.96)$ & \\
\hline $\begin{array}{l}\text { Prueba } \\
\text { Hausman }\end{array}$ & $1.8149(0.874)$ & & $1.814(0.874)$ & \\
\hline
\end{tabular}

Panel balanceado: $\mathrm{n}=21, \mathrm{~T}=12, \mathrm{~N}=252$.

Fuente: estimaciones realizadas con $R$ versión 3.5.3.

Como se reporta en la tercera columna de la tabla 3, aunque en magnitudes diferentes, los resultados del modelo que utiliza a $B S_{i t}$ como variable dependiente, son consistentes con los que utilizan $G_{i t}$, en el sentido de que los coeficientes $S_{i t}, \ln L_{i t}$ y $P_{i t}$ continúan siendo significativos. Se debe puntualizar que lo importante de estos resultados es que se confirma que un factor central en la explicación de la desigualdad salarial, en los subsectores de la manufactura, es el índice de subcontratación $S_{i t}$ (coeficiente de 39.8), que muestra un mayor coeficiente respecto a las demás variables consideradas (ver resultados de estimación con la brecha salarial en la tabla 3). Este hecho deja ver que la política de flexibilidad laboral del mercado de trabajo ha generado efectos adversos en la desigualdad salarial del sector.

Del segundo modelo, también se observa que las variables de comercio exterior $\ln C_{i t}$ y perfil tecnológico $P T_{i t}$ influyen de manera significativa a la desigualdad salarial del sector. Por un lado, se prueba la relación inversa entre la apertura comercial y la desigualdad, mayores niveles de comercio exterior $\left(\ln C_{i t}\right.$ ) reducen la brecha salarial porque el salario de los trabajadores no calificados incrementa, mientras que el de los calificados se reduce (ver Stolper-Samuelson, 1941) y, por otro lado, el perfil tecnológico $P T_{i t}$, variable proxy del cambio técnico contribuye al incremento de la brecha salarial. 


\section{Conclusiones}

En este trabajo, se analizaron los determinantes de la desigualdad salarial en la manufactura en México. Las principales conclusiones que se extraen, son las siguientes:

Las estimaciones sobre la desigualdad salarial en la industria manufacturera dejan ver que la disparidad entre los ingresos de los obreros en la industria es relativamente baja en comparación con la desigualdad del ingreso a nivel nacional. Los datos muestran que a partir de 2012, la desigualdad salarial en la manufactura se ha reducido. En relación con la desigualdad salarial al interior de los subsectores y entre sus ramas, la evidencia indica que, en 15 de los 21 subsectores, la desigualdad salarial es notoriamente baja; los 6 subsectores restantes se identifican como de alta desigualdad y destaca la industria de derivados del petróleo (324), con la mayor desigualdad.

Al estimar la función de desigualdad salarial con datos de panel, se da evidencia de que el crecimiento de la productividad del trabajo $\left(P_{i t}\right)$, de la subcontratación laboral $\left(S_{i t}\right)$ y del empleo $\left(\ln L_{i t}\right)$, aportan evidencia para considerar que son elementos que contribuyen a la explicación de la desigualdad salarial en los subsectores de la manufactura. Más aún, por la magnitud del coeficiente $S_{i t}$, mayor a los coeficientes de $P_{i t}$ y $\left(\ln L_{i t}\right)$, los resultados indicarían que las condiciones que prevalecen para la contratación del factor trabajo en los subsectores de la manufactura, vía outsourcing, asociadas a la flexibilidad laboral han sido una de las principales causas para explicar la desigualdad salarial en la manufactura. Estos resultados son congruentes con los que se obtienen al considerar como variable dependiente, a la brecha salarial en lugar del índice de Gini. Aunque en magnitudes diferentes, se confirma que un factor central en la explicación de la desigualdad salarial en los subsectores de la manufactura es la subcontratación, lo que deja ver que la política de flexibilidad laboral del mercado de trabajo, ha generado efectos adversos en la desigualdad salarial del sector. Finalmente, lo que destaca de esta segunda estimación es que el perfil tecnológico que prevalece en los subsectores de la manufactura, puede ser un factor que explique la desigualdad salarial, el coeficiente estimado, con efectos fijos, para $P T_{i t}$ es de $27.287(0.00)$.

\section{Referencias}

[1] Acemoglu, D. (2002). "Technology and the Labor Market", Journal of Economic Literature, No. 40, 7-72. 
[2] Aguayo-Tellez, E., Airola, J., Juhn, C. and Villegas-Sanchez, C. (2014). "Did Trade Liberalization Help Women? the Case of Mexico in the 1990s", New Analyses of Worker Well-Being (Research in Labor Economics, Vol. 38), Emerald Group Publishing Limited, pp. 1-35. https://doi.org/10.1108/S01479121(2013)0000038001

[3] Akerman, A., Helpman, E., Itskhoki, O., Muendler, M. y Redding, S. (2013). "Sources of Wage Inequality", American Economic Review. American Economic Association, vol. 103(3), pp. 214-219.

[4] Arceo-Gómez, Eva O. y Raymundo M. Campos-Vázquez (2014). El Trimestre Económico, vol. LXXXI (3), núm. 323, pp. 619-653.

[5] Atkinson, A. y Bourguignon, F. (2000). Handbook of Income Distribution, Vol. 1, North Holland.

[6] Atuesta Montes, B.; X. Mancero y V. Tromben Rojas (2018). Herramientas para el análisis de las desigualdades y del efecto redistributivo de las políticas públicas. Comisión Económica para América Latina y el Caribe (CEPAL). Naciones Unidas, Santiago.

[7] Banxico (2019). "Estadísticas sobre comercio exterior", Banco de México. Disponible en: https://www.banxico.org.mx/SieInternet/

[8] Bell, L. (1997). "The Impact of Minimum Wages in Mexico and Colombia", Journal of Labor Economics, 15.

[9] Bhagwati, J. (1995). "Trade and Wages: Choosing among Alternative Explanations", Economic Policy Review, Federal Reserve Bank of New York, January, 42-47.

[10] Broecke, S. (2016). "Do skills matter for wage inequality?", IZA World of Labor, February 2016.

[11] Cañonero, G. y Werner, A. (2002). "Salarios relativos y liberación del comercio en México", El Trimestre Económico, No. 273.

[12] Carbajal, Y. y Carrillo, B. (2016). "El empleo en los subsectores de la manufactura en las entidades federativas de la Región Centro de México, 1998-2014", Paradigma económico. Año 8 núm. 1, 77-105.

[13] Carbajal-Suárez, Y.; P. Mejía-Reyes y L. Rendón (2008). "Especialización y perfil tecnológico de la manufactura del Estado de México", en P. Mejía-Reyes, L. E. Del Moral y O. Rodríguez (coords.), Actividad Económica en el Estado de México. Manufactura e Industria Automotriz, Vol. II. Gobierno del Estado de México, Secretaría de Desarrollo Económico, Estado de México, pp. 69-99.

[14] Card, David y John E. DiNardo (2002). "Skill Biased Technological Change and Rising Wage Inequality: Some Problems and Puzzles", NBER. Working Paper 8769, <http://www.nber.org/papers/w8769>

[15] Castellano, S. (2010). "Desempleo y determinación de salarios en la industria manufacturera en México. Un análisis mediante paneles dinámicos". Economía mexicana. Nueva época, vol. XIX, núm. 1, primer semestre.

[16] Castro, D. y Huesca, L. (2007). "Desigualdad salarial en México: una revisión”, Papeles de Población. Vol. 13, No. 54, 225-264.

[17] Chiquiar, D., Salcedo, A. y Covarrubias, E. (2017). "Labor Market Consequences of Trade Openness and Competition in Foreign Markets". Banco de México, Documentos de Investigación. Núm. 2017-01.

[18] Contreras, D. y S. Gallegos (2007). "Descomponiendo la desigualdad salarial en América Latina: una década de cambios". Serie Estudios Estadísticos y 
Prospectivos, Núm. 59. Comisión Económica para América Latina y el Caribe.

[19] Cowell, F. (2000). "Measurement of inequality", in Atkinson A.B. and Bourguignon F. (Eds), Handbook of Income Distribution, Vol. 1, North Holland, chapter 2, 87- 166.

[20] Cragg, M. y Epelbaum, M. (1996). "Why has Wage Dispersion Grown in Mexico? Is it the Incidence of Reforms or the Growing Demand for Skills?", Journal of Development Economics, 51, 99-116.

[21] De Jesús Almonte, L. (2019). Lento crecimiento y empleo manufacturero en México. Un análisis de endogeneidad territorial. UAEM-Eón editores.

[22] De la Garza, E., Lara, S. y Torres, J. (2001). "Flexibilidad y trabajo femenino en la industria manufacturera de México". Revista Mexicana de Sociología, Vol. 63(2).

[23] Esquivel, G. y Rodríguez, J.A. (2003). "Technology, Trade and Wage Inequality in Mexico before and after NAFTA". Journal of Development Economics, 72, 543-565, December 2003.

[24] Fuentes, Noé Arón, y García Andrés, Adelaido. (2009). Jerarquización sectorial de la economía mexicana: Un enfoque de teoría de grafos. Problemas del desarrollo, 40(158), 137-159. Recuperado el 22 de diciembre de 2020, de http://www.scielo.org.mx/scielo.php?script=sci_arttext\&pid=S0301$70362009000300007 \& \operatorname{lng}=\mathrm{es} \& \ln g=\mathrm{es}$.

[25] García Andrés, A.; Fuentes, N. A., y Montes García, O. (2012). Desigualdad y polarización del ingreso en México: 1980-2008. Política y cultura, (37), 285310. Recuperado el 22 de diciembre de 2020, de http://www.scielo.org.mx/scielo.php?script=sci_arttext\&pid=S0188$77422012000100014 \& \operatorname{lng}=\mathrm{es} \&$ tlng=es.

[26] Gini, C. (1914). "Sulla misura della concentrazione e della variabilità dei caratteri", Atti del Reale Istituto Veneto di Scienze, Lettere ed Arti, 73(2), 1203-1248 (English translation: "On the measurement of concentration and variability of characters" in Metron - International Journal of Statistics, 2005, 63(1), 138).

[27] Harrison, A. y Hanson, G. (1999). "Who Gains from Trade Reform? Some Remaining Puzzles", Journal of Development Economics, Vol. 59, 125-154.

[28] Heshmati, A. (2004). "Inequalities and Their Measurement" MTT Economic Research and IZA, Discussion paper No. 1219, July.

[29] Hernández, A. (2012). "Diagnóstico del trabajo en el contexto de la globalización económica: el caso de México". Economía informa. UNAM, núm. 375, 2754.

[30] Hernández-Laos, E. (2000). "Productividad y empleo en la apertura económica de México", El Trimestre Económico. No. 265.

[31] Hoekman, B. y Winters, A. (2005). "Trade and Employment: Stylized Facts and Research Findings" United Nations Department of Economic and Social Affairs (UN/DESA) Working paper No. 7.

[32] Hsiao, C. (2003). Analysis of Panel Data, Cambridge University Press, 2nd ed., United Kingdom.

[33] Huesca Reynoso, Luis (2003). “Análisis de la polarización del ingreso de los hogares en México durante 1984-2000”, Investigación Económica, vol. LXII, núm. 246, 2003, pp. 89-123. México

[34] INEGI (2014). Censos Económicos, 2014, México: INEGI. 
[35] INEGI (2019). Encuesta Mensual de la Industria Manufacturera. INEGI. México. [36] INEGI (2019). "Estadísticas de Producto Interno Bruto y Empleo". Banco de Información Económica del INEGI en: http://www.inegi.org.mx/sistemas/bie/

[37] Krugman, P. y Lawrence, R. (1993). "Trade, Jobs and Wages" NBER Working Paper, 4478.

[38] López-Acevedo, G. (2001). "Evolution of Earnings and Rate of Returns to Education in Mexico", Working Paper, The World Bank.

[39] Lorenz, M. (1905). "Methods of measuring the concentration of wealth", Journal of the Royal Statistical Society 9, 209-219.

[40] Loría, E.; J. C. Moreno-Brid; E. Salas e I. L. Sánchez (2019). Explicación Kaldoriana del bajo crecimiento económico en México. Problemas del desarrollo, $50 \quad$ (196), 3-26. https://doi.org/10.22201/iiec.20078951e.2019.196.63506.

[41] Loría, E.; E. Ramírez Guerra y E. Salas (2015). "La Ley de Okun y la flexibilidad laboral en México: un análisis de cointegración, 1997Q3-2014Q1", Contaduría $\quad y \quad$ Administración, 60(3). doi:http://dx.doi.org/10.1016/j.cya.2015.05.012.

[42] Meza, L. (2005). "Mercados laborales locales y desigualdad salarial en México", El Trimestre Económico, No. 285, 133-178.

[43] Murillo-Villanueva, B. (2019). "Cambio técnico en la industria manufacturera en México, 2003-2008 y 2008-2012”, Paradigma económico. Año 11. Núm. 1, 41-73.

[44] Neffa, J. (2010). "La transición desde los verdaderos empleos al trabajo precario", en Trabajo, identidad y acción colectiva, Coords.: Garza, E. y J. Neffa. Plaza y Valdés editores, CLACSO y UAM Iztapalapa.

[45] Ocegueda, J. M. (2003). Análisis Kaldoriano del crecimiento en los estados de México 1980-2000. Comercio Exterior, 53 (11), 1024-1034.

[46] OIT (2012). World of Work Report. Organización Internacional del Trabajo.

[47] PNUD (2010). Informe Regional sobre Desarrollo Humano para América Latina $y$ el Caribe, 2010. Programa de Naciones Unidas para el Desarrollo. Disponible en: www.idhalc-actuarsobreelfuturo.org.

[48] Ramos, C., Alvar González, M. y Moreno, B. (2018). "Factores determinantes de la reducción de la desigualdad en la distribución de la renta en países de América Latina", Revista de la CEPAL, No. 126, 87-107.

[49] Rodríguez Pérez, R. E.; V. German-Soto y C. J. González Cuatianquis (2017). "Desigualdad salarial en el sector manufacturero de México y sus regiones, según su exposición a la apertura comercial, 2005-2015”, Equilibrio Económico, Vol. 13 (1). 43, pp. 49-76.

[50] Robins, D. (1996). "Evidence on Trade and Wages in the Developing Worlds", OECD Developing Center, Technical Paper No. 119.

[51] Sánchez-Juárez, I. L. y J. C. Moreno-Brid (2016). El reto del crecimiento económico en México. Industrias manufactureras y política industrial. Revista finanzas y politica económica, 8 (2), 271-299. http://dx.doi.org/10.14718/revfinanzpolitecon.2016.8.2.4

[52] Stolper, W. y Samuelson, P. (1941). "Protection and real wages", The Review of Economic Studies, 9(1), 58-73.

[53] Topel, R. (1994) "Regional Labor Markets and the Determinants of Wage Inequality", The American Economic Review, Vol. 84, No. 2, 17-22. 
[54] Wooldridge, J. M. (2002), Econometric Analysis of Cross Section and Panel Data, The MIT Press, United Kingdom.

[55] Zamudio, A. (2001). "La escolaridad y la distribución condicional del ingreso. Una aplicación de regresión cuantil”, El Trimestre Económico, Vol. 68, No. 269(1), pp. 39-70. 


\begin{tabular}{llll}
\multicolumn{5}{c}{$\begin{array}{c}\text { Anexo 1 } \\
\text { Panel de datos: estadísticas básicas } \\
\text { Desviación }\end{array}$} & $\begin{array}{l}\text { Coeficiente } \\
\text { estándar }\end{array}$ & $\begin{array}{l}\text { de variación } \\
\text { Media }\end{array}$ & $\begin{array}{l}\text { De61 } \\
G_{i t}\end{array}$ & 0.08 & 25.64 & 0.756 \\
$B S_{i t}$ & 23.50 & 2.89 & 1.0908 \\
$P_{i t}$ & 1.55 & 0.11 & 0.435 \\
$S_{i t}$ & 0.25 & 1.33 & 0.069 \\
$\ln C_{i t}$ & 19.16 & 1.00 & 0.089 \\
$\ln L_{i t}$ & 11.27 & 0.24 & 0.533 \\
$P T_{i t}$ & 0.46 &
\end{tabular}

Fuente: estimaciones en $\mathrm{R}$ versión 3.5.3. 